\title{
SOME UNDECIDABILITY RESULTS CONCERNING RADON MEASURES
}

BY

\author{
R. J. GARDNER AND W. F. PFEFFER
}

\begin{abstract}
We show that in metalindelof spaces certain questions about Radon measures cannot be decided within the Zermelo-Fraenkel set theory, including the axiom of choice.
\end{abstract}

0. Introduction. All spaces in this paper will be Hausdorff. Let $X$ be a space. By $\mathcal{G}$ and $C$ we shall denote, respectively, the families of all open and compact subsets of $X$. The Borel $\sigma$-algebra in $X$, i.e., the smallest $\sigma$-algebra in $X$ containing $\mathcal{G}$, will be denoted by $\mathscr{B}$. The elements of $\mathscr{B}$ are called Borel sets. A Borel measure in $X$ is a measure $\mu$ on $\mathscr{B}$ such that each $x \in X$ has a neighborhood $U \in \mathscr{B}$ with $\mu(U)<$ $+\infty$.

A Borel measure $\mu$ in $X$ is called

(i) Radon if

$$
\mu(B)=\sup \{\mu(C): C \in \mathcal{C}, C \subset B\}
$$

for each $B \in \mathscr{B}$;

(ii) regular if

$$
\mu(B)=\inf \{\mu(G): G \in \mathcal{G}, B \subset G\}
$$

for each $B \in \mathscr{B}$.

$A$ Radon space is a space $X$ in which each finite Borel measure is Radon.

The present paper will be divided into two parts, each dealing with one of the following questions.

( $\alpha$ ) Let $X$ be a locally compact, hereditarily metalindelöf space with the countable chain condition. Is $X$ a Radon space?

( $\beta$ ) Let $X$ be a metalindelöf space, and let $\mu$ be a $\sigma$-finite Radon measure in $X$. Is $\mu$ a regular measure?

We shall show that neither question can be decided within the usual axioms of set theory. The proofs are based on an alternate application of the continuum hypothesis, Martin's axiom and Ostaszewski's axiom \$ . In passing, we shall also answer a question of F. D. Tall (see [T, last paragraph in \$6]). Namely, using the continuum hypothesis and $\$$, we shall construct a locally compact, hereditarily metalindelöf space $X$ with the countable chain condition which is not weakly $\theta$-refinable.

Received by the editors July 6, 1978.

AMS (MOS) subject classifications (1970). Primary 54D20, 54G20, 54H99; Secondary 28A30, 28 A35.

Key words and phrases. Borel measure, regular measure, Radon space, metalindelöf space, continuum hypothesis, Martin's axiom, 4 .

() 1980 American Mathematical Society $0002-9947 / 80 / 0000-0203 / \$ 03.50$ 
1. Preliminaries. If $A$ is a set, we shall denote its cardinality by $|A|$. Whenever convenient we shall identify a cardinal with its initial ordinal. By $\omega$ and $\Omega$ we shall denote the first infinite and the first uncountable cardinals, respectively.

A family $\mathbb{Q}$ of subsets of a set $E$ is called, respectively, point-finite or point-countable if for each $x \in E$ the collection $\{A \in \mathbb{Q}: x \in A\}$ is finite or countable. A space $X$ is called, respectively, metacompact or metalindelöf if each open cover of $X$ has a point-finite or point-countable open refinement. A space $X$ is said to satisfy the countable chain condition (abbreviated as $\mathrm{CCC}$ ) if each disjoint family of open subsets of $X$ is countable.

The continuum hypothesis (abbreviated as $\mathrm{CH}$ ) states $2^{\omega}=\Omega$. Martin's axiom (abbreviated as MA) has several equivalent formulations; we shall use the following topological version.

MA: Let $X$ be a compact space with CCC, and let $\mathcal{H}$ be a family of open dense subsets of $X$. If $|\mathcal{H}|<2^{\omega}$, then $\cap \mathcal{H} \neq \varnothing$.

Ostaszewski's axiom will not be applied directly. Rather we shall use a space $Y$ (see 2.2) which Ostaszewski constructed assuming $\mathrm{CH}+\$$. For the formulation of \& we refer to [O].

It was proved in [ST] and [O] that both $\mathrm{MA}+\neg \mathrm{CH}$ and $\mathrm{CH}+\&$ are separately consistent with the Zermelo-Fraenkel set theory including the axiom of choice. We note that Devlin (see [D]) has proved the equivalence of $\mathrm{CH}+\&$ and Jensen's axiom $\diamond($ see $[\mathbf{J}])$.

A cardinal $\kappa$ is called measurable if there is a discrete space $X$ with $|X|=\kappa$ and a Borel measure $\nu$ in $X$ such that $\nu(X)=1$ and $\nu(\{x\})=0$ for each $x \in X$. For the properties of measurable cardinals we refer to [U] or [Di, Chapter 0, §4].

We shall occasionally mention (but never extensively use) $\theta$-refinable and weakly $\boldsymbol{\theta}$-refinable spaces. For their definitions and properties we refer to [WW] and [BL].

2. Radon spaces. The property of being a Radon space is a topological property in the sense that it is invariant under homeomorphisms. Thus one would like to characterize it topologically without referring to measures. In locally compact spaces necessary conditions have been found involving completeness properties such as $\alpha$-realcompactness (see [G, Theorem 3.5]); also considerable progress has been made in obtaining sufficient conditions by use of covering properties.

It is fairly easy to see that if a locally compact space $X$ is hereditarily Lindelöf, then it is Radon (see [H, §52]). In [K, Theorem 2], Katětov proved that if a locally compact space $X$ contains no discrete subset of measurable cardinality, then $X$ is Radon whenever $X$ is hereditarily paracompact. Paracompact was relaxed to metacompact by Haydon (see [Hy, Proposition 3.2]) and then to weakly $\boldsymbol{\theta}$-refinable by Gardner (see [G, Corollary to Theorem 6.1]). With these results at hand it is natural to investigate spaces which are hereditarily metalindelöf.

2.1. Theorem. Assume $M A+\neg C H$. Let $X$ be locally compact and hereditarily metalindelöf. If $X$ satisfies $C C C$, or if $X$ satisfies $C C C$ locally and contains no discrete subset of measurable cardinality, then $X$ is a Radon space. 
Proof. By [T, Theorems 6.9 or 6.10], each open subset of $X$ is, respectively, Lindelöf or paracompact. The theorem follows from [P, (18.15)] or [K, Theorem 2], respectively.

We do not know whether the previous theorem remains correct if the locally CCC condition is omitted. To show that assuming $\mathrm{MA}+\neg \mathrm{CH}$ is essential, we shall need a result of Ostaszewski, and an unpublished result of Kunen, who kindly provided us with a preprint.

2.2. Proposition [O]. Assume $\mathrm{CH}+\$$. Then there is a countably compact, noncompact space $Y$ with $|Y|=\Omega$ and such that it is perfectly normal, locally countable, locally compact, and hereditarily separable.

2.3. Proposition [Ku]. Assume $\mathrm{CH}$. Then there is a nonseparable, compact space $Z$ with $|Z|=\Omega$ and such that it is hereditarily Lindelöf, totally disconnected, and has no isolated points. Moreover, there is a Radon measure $\nu$ in $Z$ such that $\nu(Z)=1$ and $\nu(B)=0$ if and only if $B \subset Z$ is a nowhere dense Borel set.

Throughout this section we shall assume $\mathrm{CH}+\&$, and we shall construct a space $X_{0} \subset Y \times Z$.

As $Y$ is locally countable we can cover it by a collection $\left\{U_{\alpha}: \alpha<\Omega\right\}$ of nonempty, countable, open sets. Since $Z$ is hereditarily Lindelöf, it is first countable (see [AU, Chapter II, Theorems 6, 4]). By [T, Theorem 3.27] there is a point-countable family $\left\{V_{\alpha}: \alpha<\Omega\right\}$ of distinct, nonempty, open subsets of $Z$. We set $X_{0}=U\left\{U_{\alpha} \times V_{\alpha}: \alpha<\Omega\right\}$ and we shall give it the relative topology from $Y \times Z$. Clearly, $X_{0}$ is a first countable, locally compact space. If $A \subset X_{0}$ and $y \in Y$, let $A_{y}=\{z \in Z:(y, z) \in A\}$.

\subsection{Proposition. The space $X_{0}$ is hereditarily metalindelöf with CCC.}

Proof. Choose $A \subset X_{0}$. If $\mathscr{Q}$ is an open cover of $A$, let $\mathscr{T}$ be an open cover of $A$ which is a common refinement of $\mathcal{Q}$ and $\left\{U_{\alpha} \times V_{\alpha}: \alpha<\Omega\right\}$. Since $U_{\alpha}$ is countable and $Z$ is hereditarily Lindelöf, there is a subcover $\mathscr{} \subset \mathcal{V}$ of $A$ such that $\left|\left\{W \in W: W \subset U_{\alpha} \times V_{\alpha}\right\}\right| \leqslant \omega$ for each $\alpha<\Omega$. The point countability of $W$ follows from that of $\left\{V_{\alpha}: \alpha<\Omega\right\}$. Now suppose that $\mathcal{H}$ is an uncountable disjoint family of open subsets of $X_{0}$. If $D$ is a countable dense subset of $Y$, then for some $y \in D$ the family $\left\{H_{y}: H \in \mathcal{C}\right\}$ is uncountable. However, this is impossible as $Z$ is hereditarily Lindelöf.

Our next objective is to define a finite Borel measure in $X_{0}$ which is not Radon.

By [GJ, 1.4 and 5.9], $Y$ is not realcompact. Because $Y$ is perfectly normal, there is a two-valued Borel measure $\lambda$ in $Y$ such that $\lambda(Y)=1$ and $\lambda(\{y\})=0$ for each $y \in Y$. If $A$ and $B$ are sets, let $A \triangle B=(A-B) \cup(B-A)$. Denote by $\Re$ the family of those $M \subset Y \times Z$ for which there are Borel sets $M_{Y} \subset Y$ and $M_{Z} \subset Z$ such that $\lambda\left(M_{Y}\right)=1$ and for each $y \in M_{Y}, M_{y}$ is a Borel subset of $Z$ and $\nu\left(M_{y} \Delta M_{Z}\right)=0$. If $M \in \mathscr{T}$, let $\mu(M)=\nu\left(M_{Z}\right)$.

In several lemmas we shall prove that $\mathscr{T}$ is a $\sigma$-algebra in $Y \times Z$ containing closed sets and that $\mu$ is a measure on $\mathfrak{R}$. 
2.5. LemMA. The family $\mathfrak{N}$ is a $\sigma$-algebra in $Y \times Z$ and $\mu$ is a measure on $\mathfrak{N}$.

Proof. Clearly, $Y \times Z \in \mathfrak{N}$. Let $A \in \mathfrak{N}$ and let $B=Y \times Z-A$. Setting $B_{Y}=A_{Y}$ and $B_{Z}=Z-A_{Z}$, we see that $B \in \Re$. If $A_{n} \in \Re, n=1,2, \ldots$, and $A=\cup_{n=1}^{\infty} A_{n}$, let $A_{Y}=\bigcap_{n=1}^{\infty}\left(A_{n}\right)_{Y}$ and $A_{Z}=\cup_{n=1}^{\infty}\left(A_{n}\right)_{Z}$. Since $\lambda\left(A_{Y}\right)=1$, it follows that $A \in \mathfrak{R}$. Moreover, if $A_{n}$ are disjoint, then $\nu\left[\left(A_{n}\right)_{Y} \cap\left(A_{m}\right)_{Y}\right]=0$ whenever $n \neq m$. Consequently,

$$
\mu(A)=\nu\left(A_{Y}\right)=\sum_{n=1}^{\infty} \nu\left[\left(A_{n}\right)_{Y}\right]=\sum_{n=1}^{\infty} \mu\left(A_{n}\right) .
$$

2.6. Lemma. Let $F$ be a closed subset of $Y \times Z$. Then $\Gamma_{\varepsilon}=\left\{y \in Y: \nu\left(F_{y}\right)>\varepsilon\right\}$ is a closed subset of $Y$ for each real number $\varepsilon$.

Proof. Let $\left\{y_{n}\right\}_{n=1}^{\infty}$ be a sequence in $\Gamma_{\varepsilon}$ converging to a $y \in Y$. Let $H=$ $\bigcap_{k=1}^{\infty} \cup_{n=k}^{\infty} F_{y_{n}}$ and choose $z \in H$. Then there is a subsequence $\left\{y_{n}^{\prime}\right\}$ of $\left\{y_{n}\right\}$ such that $\left(y_{n}^{\prime}, z\right) \in F$ for $n=1,2, \ldots$ Since $F$ is closed and $\left(y_{n}^{\prime}, z\right) \rightarrow(y, z)$, we have $z \in F_{y}$. Thus $H \subset F_{y}$ and $y \in \Gamma_{e}$; for $\nu\left(F_{y}\right)>\nu(H)>\varepsilon$. Because $Y$ is first countable, $\Gamma_{e}$ is closed.

2.7. Lemma. Let $F \subset Y \times Z$ be closed. Then there is an $r>0$ and $a \Gamma \subset Y$ such that $\lambda(\Gamma)=1$ and $\lambda\left(F_{y}\right)=r$ for each $y \in \Gamma$.

Proof. Let $\Gamma_{\varepsilon}$ be the closed subset of $Y$ defined in Lemma 2.6, and let $r=\sup \left\{\varepsilon: \lambda\left(\Gamma_{e}\right)=1\right\}$. Since $\Gamma_{0}=Y, r>0$. If

$$
\Gamma=\bigcap_{n=1}^{\infty} \Gamma_{r-1 / n}-\bigcup_{n=1}^{\infty} \Gamma_{r+1 / n},
$$

then $\lambda(\Gamma)=1$ and $\nu\left(F_{y}\right)=r$ for each $y \in \Gamma$.

2.8. Lemma. If $F$ is a closed subset of $Y \times Z$, then $F \in \Re$.

Proof. Let $r$ and $\Gamma$ be as in Lemma 2.7. Choose $\varepsilon>0$, and for each $y \in \Gamma$ find a compact set $C \subset Z-F_{y}$ so that $\nu\left(Z-F_{y}\right)<\nu(C)+\varepsilon / 2$. For each $z \in Z-F_{y}$ there are open neighborhoods $U(z)$ and $V(z)$ of $y$ and $z$, respectively, such that $U(z) \times V(z) \subset X-F$. Since the $V(z)$ 's cover $Z-F_{y}$, finitely many of them, say $V\left(z_{1}\right), \ldots, V\left(z_{n}\right)$, cover $C$. Setting $G(y)=\cap_{k=1}^{n} U\left(z_{k}\right)$, we have $F_{y^{\prime}} \subset Z-C$ for each $y^{\prime} \in G(y)$. If $y^{\prime} \in G(y) \cap \Gamma$, then

$$
\begin{aligned}
\nu(Z-C) & >\nu\left(F_{y} \cup F_{y^{\prime}}\right)=2 \nu\left(F_{y}\right)-\nu\left(F_{y} \cap F_{y^{\prime}}\right) \\
& >2[\nu(Z-C)-\varepsilon / 2]-\nu\left(F_{y} \cap F_{y^{\prime}}\right),
\end{aligned}
$$

and consequently,

$$
\nu\left(F_{y} \Delta F_{y^{\prime}}\right)=\nu\left(F_{y} \cup F_{y^{\prime}}\right)-\nu\left(F_{y} \cap F_{y^{\prime}}\right)<\varepsilon .
$$

Since $Y$ is hereditarily separable, $\Gamma$ has a countable dense subset $\left\{y_{n}: n=\right.$ $1,2, \ldots\}$. The sets

$$
\Gamma_{n}=\left\{y \in \Gamma: y \in G\left(y_{n}\right) \text { or } y_{n} \in G(y)\right\}
$$

are open in $\Gamma$ and $\Gamma=\cup_{n=1}^{\infty} \Gamma_{n}$. Hence $\lambda\left(\Gamma_{N}\right)=1$ for some integer $N \geqslant 1$. Letting $\Lambda_{\varepsilon}=\Gamma_{N}$ and $H_{e}=F_{y_{N}}$, we obtain $\nu\left(F_{y} \Delta H_{e}\right)<\varepsilon$ for each $y \in \Lambda_{e}$. Now let 
$F_{Y}=\cap_{n=1}^{\infty} \Lambda_{1 / n}$. Then $\lambda\left(F_{Y}\right)=1$, and we set $F_{Z}=F_{y_{0}}$ for some $y_{0} \in F_{Y}$. If $y \in F_{Y}$, then

$$
\nu\left(F_{y} \Delta F_{z}\right) \leqslant \nu\left(F_{y} \Delta H_{1 / n}\right)+\nu\left(H_{1 / n} \Delta F_{y_{0}}\right)<2 / n
$$

for $n=1,2, \ldots$ Thus $\nu\left(F_{y} \Delta F_{Z}\right)=0$ for each $y \in F_{Y}$ and $F \in \mathfrak{T}$.

Denoting by $\bar{\lambda}$ the completion of the measure $\lambda$ in $Y$, we obtain the following corollary.

2.9. Corollary. There is a Borel measure $\mu$ in $Y \times Z$ such that

$$
\mu(B)=\int_{Y} \nu\left(B_{y}\right) d \bar{\lambda}(y)
$$

for each Borel set $B \subset Y \times Z$.

Denote by $\mu_{0}$ the restriction of the measure $\mu$ from Corollary 2.9 to the Borel subsets of $X_{0}$.

2.10. TheOREM. $0<\mu_{0}\left(X_{0}\right) \leqslant 1$ and $\mu_{0}\left(U_{\alpha} \times V_{\alpha}\right)=0$ for each $\alpha<\Omega$.

Proof. Clearly, $\mu_{0}\left(X_{0}\right) \leqslant \mu(Y \times Z)=1$. Since the $U_{\alpha}$ 's are countable $\mu_{0}\left(U_{\alpha} \times\right.$ $\left.V_{\alpha}\right)=0$ for each $\alpha<\Omega$. The sets

$$
A_{n}=\bigcup\left\{U_{\alpha}: \alpha<\Omega, \nu\left(V_{\alpha}\right) \geqslant 1 / n\right\}, \quad n=1,2, \ldots,
$$

form an open cover of $Y$. Thus there is an integer $N>1$ with $\lambda\left(A_{N}\right)=1$. Letting $B=\cup\left\{U_{\alpha} \times V_{\alpha}: U_{\alpha} \subset A_{N}\right\}$, we have $\mu_{0}\left(X_{0}\right)>\mu_{0}(B)>1 / N$.

2.11. Corollary. The space $X_{0}$ is not Radon.

2.12. Corollary. The space $X_{0}$ is not weakly $\theta$-refinable.

Proof. Since $\left|X_{0}\right|=\Omega, X_{0}$ contains no subset of measurable cardinality (see [Di, Theorem 0.4.17]). The corollary follows from [G, Theorem 4.1 and the paragraph following the corollary to Theorem 4.3].

2.13. Remarks. ( $\alpha$ ) Clearly, the measure $\mu$ from Corollary 2.9 is an extension of the product measure $\bar{\lambda} \times \nu$. We claim that this extension is proper. Indeed, since the family $\left\{V_{\alpha}: \alpha<\Omega\right\}$ is point-countable, the set $X_{0}^{z}=\left\{y \in Y:(y, z) \in X_{0}\right\}$ is countable for each $z \in Z$. Thus $\int_{Z} \bar{\lambda}\left(X_{0}^{z}\right) d \nu(z)=0$, and it follows that $X_{0}$ is not $\bar{\lambda} \times \nu$ measurable (see $[H, \S 35$, Theorem A]).

( $\beta$ ) It is noteworthy that for the construction of the measure $\mu$ we used only the following facts:

(i) $\lambda$ is a finite, two-valued Borel measure in $Y$;

(ii) $\nu$ is a finite Radon measure in $Z$;

(iii) $Y$ is first countable and hereditarily separable.

We do not know whether the two-valuedness of $\lambda$ is essential.

( $\gamma$ ) Applying [GG, Lemma 4], we can also construct the measure $\mu$ if $Y$ is the space of all countable ordinals and $\lambda$ is the Dieudonné measure in $Y$ (see $[H, \S 52$, Exercise 10]). In this case we do not need \&, but the space $X_{0}$ will satisfy CCC only locally. 
3. Regular measures. Clearly, each finite Radon measure is regular, and it is easy to construct a nonregular Radon measure which is not $\sigma$-finite (see [GP, Example 6]). If a Radon measure in $X$ is $\sigma$-finite, it is regular whenever $X$ is metacompact, and metacompact cannot be replaced by $\theta$-refinable (see [GP, Theorem 1 and Example 7]). Thus again, we would like to know what happens if $X$ is metalindelöf.

We shall begin with a substantially simplified and more transparent proof of [GP, Theorem 1].

Following [S, p. 43], we shall call a Borel measure $\mu$ in $X$ moderated whenever there are open sets $G_{n} \subset X$ such that $\mu\left(G_{n}\right)<+\infty, n=1,2, \ldots$, and $\cup_{n=1}^{\infty} G_{n}$ $=X$.

3.1. Proposition. A o-finite Radon measure $\mu$ in $X$ is regular if and only if it is moderated.

Proof. By the $\sigma$-finiteness of $\mu$ there are $X_{n} \in \mathscr{B}$ such that $\mu\left(X_{n}\right)<+\infty$, $n=1,2, \ldots$, and $\cup_{n=1}^{\infty} X_{n}=X$. If $\mu$ is regular, there are $G_{n} \in \mathcal{G}$ such that $X_{n} \subset G_{n}$ and $\mu\left(G_{n}\right)<+\infty, n=1,2, \ldots$ It follows that $\mu$ is moderated. Conversely, if $\mu$ is moderated, we may assume that $X_{n} \in \mathcal{G}, n=1,2, \ldots$ Let $A \in \mathscr{B}$ and let $\varepsilon>0$. Since $\mu$ restricted to $\left\{B \cap X_{n}: B \in \mathscr{B}\right\}$ is a finite Radon measure in $X_{n}$, it is regular in $X_{n}$. Thus for $n=1,2, \ldots$, there are open subsets $G_{n}$ of $X_{n}$ with $\mu\left(G_{n}\right)<\mu\left(A \cap X_{n}\right)+\varepsilon 2^{-n}$. Setting $G=\bigcup_{n=1}^{\infty} G_{n}$, we have

$$
\mu(G-A) \leqslant \mu\left[\bigcup_{n=1}^{\infty}\left(G_{n}-A \cap X_{n}\right)\right] \leqslant \sum_{n=1}^{\infty} \mu\left(G_{n}-A \cap X_{n}\right)<\varepsilon .
$$

Because each $X_{n}$ is open in $X$, so is $G$, and the regularity of $\mu$ in $X$ follows.

3.2. LEMMA. Let $(M, \mathfrak{R}, \mu)$ be an abstract measure space with a $\sigma$-finite measure $\mu$, and let $Q \subset \mathfrak{N}$ be a point-finite family. Then $\mu(A)=0$ for all but countably many $A \in \mathbb{Q}$.

Proof. Since each $\sigma$-finite measure is a countable sum of finite measures, it suffices to prove the lemma for $\mu$ finite. If $\mu(A)>0$ for uncountably many $A \in \mathcal{Q}$, then there is an $\varepsilon>0$ and distinct $A_{n} \in \mathbb{Q}$ such that $\mu\left(A_{n}\right)>\varepsilon$ for $n=1,2, \ldots$ Letting $A=\cap \bigcap_{k=1}^{\infty} \cup_{n=k}^{\infty} A_{n}$, we have $\mu(A)>\varepsilon$. In particular, $A \neq \varnothing$ which contradicts the point-finiteness of $\mathbb{Q}$.

3.3. Theorem [GP, Theorem 1]. Let $\mu$ be a $\sigma$-finite Radon measure in $X$. If $X$ is metacompact, then $\mu$ is regular.

Proof. For each $x \in X$ choose an open neighborhood $U(x)$ of $x$ with $\mu[U(x)]<$ $+\infty$. Let $\mathscr{W}$ be an open point-finite refinement of $\{U(x): x \in X\}$. By Lemma 3.2, there is a countable family $\mho_{0} \subset \mathcal{W}$ such that $\mu(V)=0$ for each $V \in W-\mho_{0}$. Let $\mho_{0}=\left\{V_{1}, V_{2}, \ldots\right\}$ and let $V_{0}=\cup\left(\mho-\mho_{0}\right)$. Clearly, $\mu\left(V_{n}\right)<+\infty, n=$ $1,2, \ldots$, and since $\mu$ is Radon, $\mu\left(V_{0}\right)=0$. Thus $\mu$ is moderated and the theorem follows from Proposition 3.1.

If $\mu$ is a Radon measure in $X$, we let

$$
\operatorname{supp} \mu=X-\bigcup\{G \in \mathcal{G}: \mu(G)=0\}
$$


and we shall call this set the support of $\mu$. Clearly, supp $\mu$ is a closed set, and $\mu(G)>0$ for each $G \in \mathcal{G}$ with $G \cap \operatorname{supp} \mu \neq \varnothing$. Since $\mu$ is Radon, we also have $\mu(X-\operatorname{supp} \mu)=0$.

For studying the regularity of Radon measures in metalindelöf spaces we shall need a lemma similar to [S, Theorem 13, p. 46].

3.4. Lemma. Let $\mu$ be a Radon measure in $X$. Then there is a disjoint family $\mathscr{D}$ of nonempty compact sets such that

(i) if $D \in \mathscr{D}$ and $D \cap G \neq \varnothing$ for $a G \in \mathcal{G}$, then $\mu(D \cap G)>0$;

(ii) if $B \in \mathscr{B}$, then $\mu(B)=\Sigma\{\mu(B \cap D): D \in \mathscr{D}\}$.

Proof. By Zorn's lemma there is a maximal disjoint family $\mathscr{D}$ of nonempty compact sets satisfying condition (i). Since $\mathscr{D}$ is disjoint,

$$
\mu(B) \geqslant \sum\{\mu(B \cap D): D \in \mathscr{D}\}
$$

for each $B \in \mathscr{B}$. Suppose that $B \in \mathscr{B}$ and $B \cap \cup \mathscr{D}=\varnothing$. If $\mu(B)>0$ then there is a $C \in \mathcal{C}$ with $C \subset B$ and $\mu(C)>0$. Let $D_{0}$ be the support of $\mu$ restricted to $\{A \cap C: A \in \mathscr{B}\}$. Adding $D_{0}$ to $\mathscr{D}$, we obtain a contradiction to the maximality of $\mathscr{D}$. If $C \in \mathcal{C}$, we can cover $C$ by finitely many sets $G \in \mathcal{G}$ with $\mu(G)<+\infty$. By the construction of $\mathscr{D}$, each $G \in \mathcal{G}$ with $\mu(G)<+\infty$ intersects only countably many $D$ 's from $\mathscr{D}$. Thus there is a countable family $\mathscr{D}_{0} \subset \mathscr{D}$ such that $(C-$ $\left.\cup \mathscr{D}_{0}\right) \cap \cup \mathscr{D}=\varnothing$. It follows that

$$
\mu(C)=\mu\left(C \cap \cup \mathscr{D}_{0}\right)=\sum\{\mu(C \cap D): D \in \mathscr{D}\} .
$$

If $B \in \mathscr{B}$, then

$$
\mu(C)=\sum\{\mu(C \cap D): D \in \mathscr{D}\}<\sum\{\mu(B \cap D): D \in \mathscr{D}\}
$$

for each $C \in \mathcal{C}$ with $C \subset B$. Since $\mu$ is Radon,

$$
\mu(B) \leqslant \sum\{\mu(B \cap D): D \in \mathscr{D}\} .
$$

Any family $\mathscr{D}$ satisfying the conditions of Lemma 3.4 is called a concassage of $\mu$.

3.5. Corollary. Let $\mu$ be a Radon measure in $X$, and let $\mathscr{D}$ be a concassage of $\mu$. Then $\mu$ is $\sigma$-finite if and only if $\mathscr{D}$ is countable.

3.6. TheOREM. Assume $M A+\neg C H$. Let $\mu$ be a $\sigma$-finite Radon measure in $X$. If $X$ is metalindelöf, then $\mu$ is regular.

Proof. For each $x \in X$ choose an open neighborhood $U(x)$ of $x$ with $\mu[U(x)]<$ $+\infty$. Let $\mathcal{T}$ be an open point-countable refinement of $\{U(x): x \in X\}$. By Lemma 3.4 and Corollary 3.5, there is a countable concassage $\mathscr{D}$ of $\mu$. Let

$$
V_{0}=\bigcup\{V \in \mathscr{V}: V \cap \bigcup \mathscr{D}=\varnothing\}, \quad \mathscr{V}_{1}=\{V \in \mathscr{V}: V \cap \bigcup \mathscr{D} \neq \varnothing\} .
$$

If $\mathscr{V}_{1}$ is uncountable, then there is a $D \in \mathscr{D}$ such that $\left\{V \in \mathscr{V}_{1}: V \cap D \neq \varnothing\right\}$ is also uncountable. Thus $\left\{V \cap D: V \in \mathfrak{V}_{1}\right\}$ is an uncountable, point-countable collection of open subsets of a compact set $D$. Since $D \in \mathscr{D}, D$ satisfies CCC. Using [T, Corollary 3.7], we obtain a contradiction. Hence $\mathfrak{V}_{1}$ is countable. Clearly, 
$\mu(V)<+\infty$ for each $V \in \mathscr{V}_{1}$, and by Lemma $3.4, \mu\left(V_{0}\right)=0$. The theorem follows from Proposition 3.1.

3.7. TheOREM. Assume CH. Then there is a totally disconnected, first countable, locally compact space $X$ with $|X|=\Omega$ and such that it is hereditarily metalindelöf, hereditarily $\theta$-refinable, and has no isolated points. Moreover, there is a $\sigma$-finite, nonregular, Radon measure $\mu$ in $X$ such that supp $\mu=X$ and $\mu(\{x\})=0$ for each $x \in X$.

Proof. Let the space $Z$ and the measure $\nu$ in $Z$ be as in Proposition 2.3. For nonnegative integers $k$ and $n$, let $q_{k, n}=\left(k 2^{-n}, 2^{-n}\right)$. Set

$$
Q=\left\{q_{k, n}: k=0, \ldots, 2^{n} ; n=0,1, \ldots\right\}
$$

and $X=[0,1] \cup(Q \times Z)$. We shall write $Z_{k, n}$ for $\left\{q_{k, n}\right\} \times Z$, and we shall define a topology in $X$ by specifying the neighborhood bases. If $x=\left(q_{k, n}, z\right)$ belongs to $Q \times Z$, the neighborhood base at $x$ is given by the sets $\left\{q_{k, n}\right\} \times V$ where $V$ is a neighborhood of $z$ in $Z$. Some more work is needed to define the neighborhood base at $x \in[0,1]$. As we have already shown in the paragraphs following Proposition 2.3 , there is a point-countable family $\mathcal{H}$ of nonempty open subsets of $Z$ with $|\mathcal{H}|=\Omega$. Since $Z$ is totally disconnected, we may assume that $\mathcal{H}$ consists of clopen (short for "closed and open") sets. Indeed, choosing a nonempty clopen set $G_{H} \subset H$ for each $H \in \mathcal{K}$, the family $\left\{G_{H}: H \in \mathcal{C}\right\}$ is point-countable and $\mid\left\{G_{H}\right.$ : $H \in \mathcal{H}\} \mid=\Omega$; for by the point-countability of $\mathcal{H}$, the map $H \mapsto G_{H}$ is countableto-one. We may also assume that there is an $r>0$ such that $\nu(H)>r$ for each $H \in \mathcal{H}$. By $\mathrm{CH}$, there is a bijection $x \mapsto H(x)$ between $[0,1]$ and $\mathcal{H}$. We shall write $H_{k, n}(x)$ for $\left\{q_{k, n}\right\} \times H(x)$. Now the neighborhood base at $x \in[0,1]$ is given by the sets

$$
U(x, \varepsilon)=\{x\} \cup \bigcup\left\{H_{k, n}(x): q_{k, n} \in Q \text { and }\left|k 2^{-n}-x\right|<2^{-n-1}<\varepsilon\right\}
$$

where $\varepsilon>0$. To facilitate a geometric visualization of the set $U(x, \varepsilon)$, we note that the projection of $U(x, \varepsilon)-\{x\}$ to $Q$ consists of those points $q_{k, n}$ which lie inside the open wedge in $[0,1] \times[0,1]$ with the vertex at $(x, 0)$, the height equal to $\varepsilon / 2$, and the slopes of the sides equal to \pm 2 .

It is easy to see that with this topology, $X$ is a first countable, locally compact, totally disconnected, Hausdorff space with no isolated points. Let $E \subset X$. We shall show that $E$ is metalindelöf and $\theta$-refinable. Since $Q \times Z$ is hereditarily Lindelöf, it is also hereditarily paracompact. Thus each open cover of $E$ has an open refinement

$$
\mathscr{U}=\mathfrak{V} \cup\left\{U\left(x, \varepsilon_{x}\right): x \in[0,1] \cap E\right\}
$$

where $\mathscr{V}$ is a point-finite family of subsets of $Q \times Z$. Suppose that some $x_{0} \in X$ is contained in uncountably many $U\left(x, \varepsilon_{x}\right)$. Then there are integers $k$ and $n, 0<k<$ $2^{n}$, such that $x_{0}$ is contained in $H_{k, n}(x)$ for uncountably many $x \in[0,1]$. However, this is impossible as $\left\{H_{k, n}(x): x \in[0,1]\right\}$ is a point-countable family in $Z_{k, n}$. Consequently, $\mathcal{Q}$ is point-countable. Letting 


$$
\begin{gathered}
\mathscr{U}_{n}=\left\{U\left(x, \varepsilon_{x} / n\right): x \in[0,1] \cap E\right\}, \quad G_{n}=\cup \mathscr{Q}_{n}, \\
\mathscr{V}_{n}=\left\{V \in \mathscr{V}: V \cap G_{n}=\varnothing\right\} \cup \mathscr{U}_{n},
\end{gathered}
$$

$n=1,2, \ldots$, we see immediately that $\cup_{n=1}^{\infty} \mathscr{V}_{n}$ is a $\theta$-refinement of $\mathcal{U}$.

Denote by $\nu_{k, n}$ the Radon measure in $Z_{k, n}$ induced by $\nu$ via the natural homeomorphism between $Z_{k, n}$ and $Z$. If $B$ is a Borel subset of $X$, let

$$
\mu(B)=\sum_{n=0}^{\infty} 2^{-n} \sum_{k=0}^{2^{n}} \nu_{k, n}\left(B \cap Z_{k, n}\right) \text {. }
$$

Because for each integer $n \geqslant 0$, the set $U(x, \varepsilon)$ intersects at most one $Z_{k, n}$, we have $\mu[U(x, \varepsilon)]<2$. It follows that $\mu$ is a Borel measure in $X$. Since each $\nu_{k, n}$ is a finite Radon measure in $Z_{k, n}$, it is easy to see that $\mu$ is a $\sigma$-finite Radon measure in $X$. Also supp $\mu=X$, for $Q \times Z$ is dense in $X$, and supp $\nu_{k, n}=Z_{k, n}, k=0, \ldots, 2^{n}$, $n=0,1, \ldots$ Clearly, $\mu(\{x\})=0$ for each $x \in X$.

Let $G$ be an open subset of $X$ containing $[0,1]$. For each $x \in[0,1]$ there is a $U\left(x, \varepsilon_{x}\right) \subset U$. Consider the natural topology of $[0,1]$. By the Baire category theorem, there is a nonempty open interval $J \subset[0,1]$ and an $\varepsilon>0$ such that the set $\left\{x \in J: \varepsilon_{x}>2 \varepsilon\right\}$ is dense in $J$. Thus $H_{k, n}(x) \subset G$ whenever $q_{k, n} \in J \times[0, \varepsilon]$. Choose an integer $N>1$ so that

$$
\left|\left\{k: q_{k, N} \in J \times[0, \varepsilon]\right\}\right|>2 .
$$

Then

$$
\left|\left\{k: q_{k, N+n} \in J \times[0, \varepsilon]\right\}\right|>2^{n},
$$

$n=0,1, \ldots$, and it follows that

$$
\mu(G) \geqslant \sum_{n=0}^{\infty} 2^{-(N+n)}\left(2^{n} r\right)=+\infty .
$$

As $\mu([0,1])=0$, the measure $\mu$ is not regular.

\section{REFERENCES}

[AU] P. S. Alexandroff and P. S. Urysohn, Mémoire sur les espaces topologique compacts, Verh. Konink. Nedrl. Akad. Wetensch. Afd. Natuurk. Amsterdam 14 (1929), 1-96.

[BL] H. R. Bennet and D. J. Lutzer, A note on weak $\theta$-refinability, General Topology and Appl. 2 (1972), 49-54.

[D] K. J. Devlin, Variations on $\diamond$, J. Symbolic Logic 44 (1979), 51-58.

[Di] M. A. Dickmann, Large infinitary languages, North-Holland, Amsterdam, 1975.

[G] R. J. Gardner, The regularity of Borel measures and Borel measure-compactness, Proc. London Math. Soc. 30 (1975), 95-113.

[GG] G. Gruenhage and R. J. Gardner, Completeness and weak covering properties, and measure-compactness, J. London Math. Soc. 18 (1978), 316-324.

[GJ] L. Gillman and M. Jerison, Rings of continuous functions, Van Nostrand, Princeton, N. J., 1960.

[GP] G. Gruenhage and W. F. Pfeffer, When inner regularity of Borel measures implies regularity, J. London Math. Soc. 17 (1978), 165-171.

[H] P. R. Halmos, Measure theory, Van Nostrand, Princeton, N. J., 1950.

[Hy] R. Haydon, On compactness in spaces of measures and measure compact spaces, Proc. London Math. Soc. 29 (1974), 1-16.

[J] R. B. Jensen, The fine structure of the constructable universe, Ann. Math. Logic 4 (1972), 229-308.

[K] M. Katètov, Measures in fully normal spaces, Fund. Math. 38 (1951), 73-84.

[Ku] K. Kunen (to appear). 
[O] A. J. Ostaszewski, On countably compact, perfectly normal spaces, J. London Math. Soc. 14 (1976), 505-516.

[P] W. F. Pfeffer, Integrals and measures, Dekker, New York, 1977.

[S] L. Schwartz, Radon measures on arbitrary topological spaces and cylindrical measures, Oxford Univ. Press, London, 1973.

[ST] R. M. Solovay and S. Tennenbaum, Iterated Cohen extensions and Souslin's problem, Ann. of Math. (2) 94 (1971), 201-245.

[T] F. D. Tall, The countable chain condition versus separability-applications of Martin's axiom, General Topology and Appl. 4 (1974), 315-339.

[U] S. Ulam, Zur Masstheorie in der allgemeinen Mengenlehre, Fund. Math. 16 (1930), 140-150.

[WW] J. M. Worrel and H. H. Wicke, Characterizations of developable topological spaces, Canad. J. Math. 17 (1965), 820-830.

Department of Mathematics, University of California, Davis, California 95616 (Curtent address of W. F. Pfeffer)

Current address (R. J. Gardner): Department of Mathematics, Auburn University, Auburn, Alabama 36830 